\title{
Correction: RCP induces Slug expression and cancer cell invasion by stabilizing $\beta 1$ integrin
}

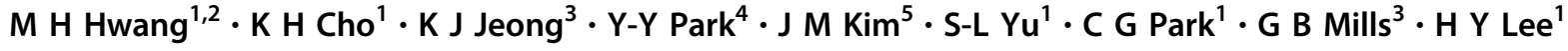

Published online: 24 January 2019

(C) Springer Nature Limited 2019

\section{Correction to: Oncogene;}

https://doi.org/10.1038/onc.2016.277; Published online 23 Feb 2017

Following the publication of this article the authors noted that images were inadvertently duplicated in Fig. 1b. The corrected Fig. 1 can be found in the associated Correction. The conclusions of this paper are not affected. The authors sincerely apologize for this error. This error has not been corrected in the HTML or PDF of the original Article.

These authors contributed equally: Min Ha Hwang, Kyung Hwa Cho, Kang Jin Jeong

The original article can be found online at https://doi.org/10.1038/onc. 2016.277

H Y Lee

hoi@konyang.ac.kr

1 Department of Pharmacology, College of Medicine, Konyang University, Daejeon, Korea

2 Department of Breast Medical Oncology, The University of Texas MD Anderson Cancer Center, Houston, TX, USA

3 Department of Systems Biology, The University of Texas MD Anderson Cancer Center, Houston, TX, USA

4 Asan Institute for Life Sciences, Asan Medical Center, Department of Medicine, University of Ulsan College of Medicine, Seoul, Korea

5 Cancer Research Institute, Regional Cancer Center and Infection Signaling Network Research Center, Chungnam National University School of Medicine, Daejeon, Korea 

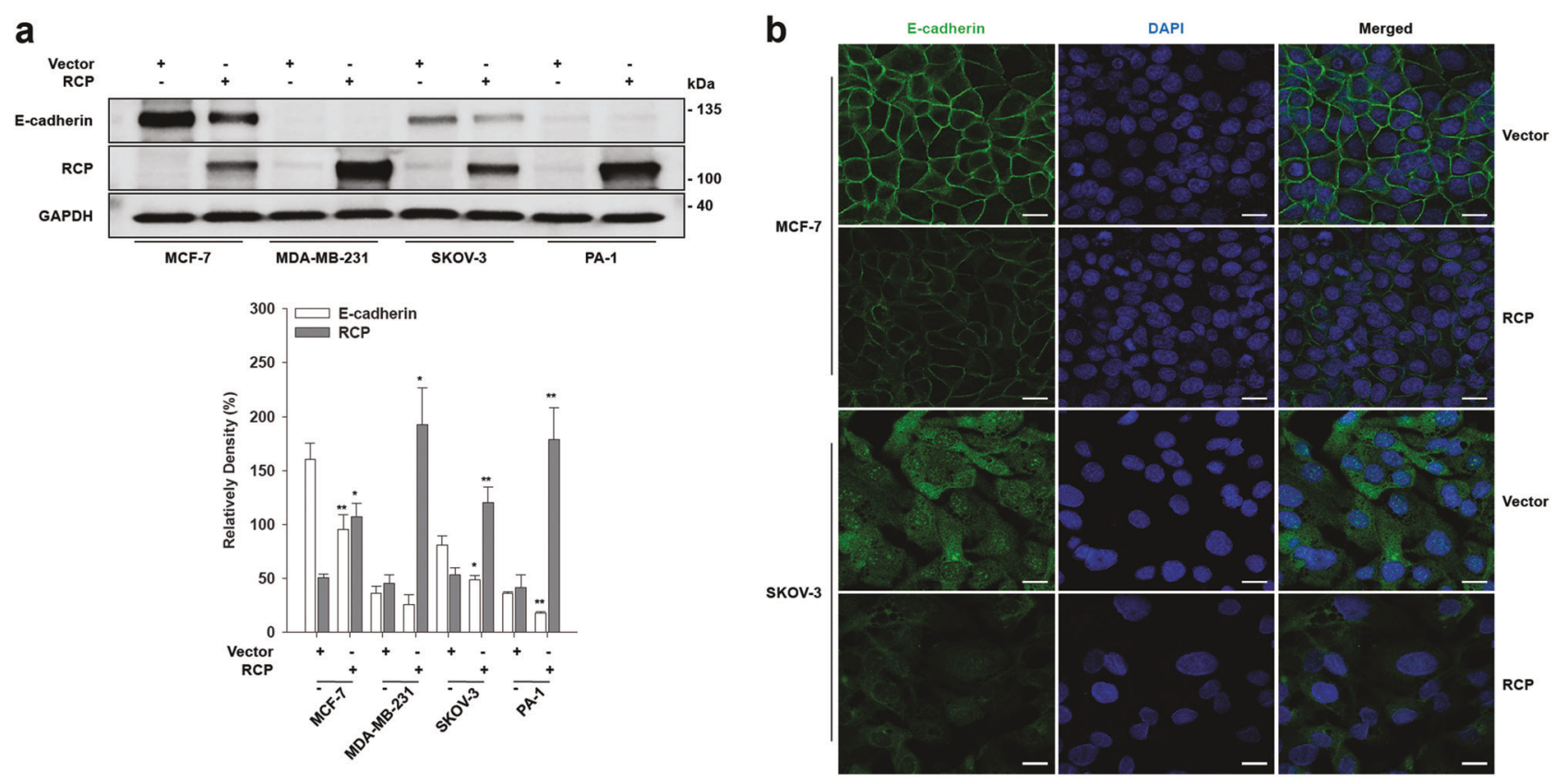

Fig. 1 RCP downregulates E-cadherin expression. a The cells were transfected with indicated vectors for $48 \mathrm{~h}$. The cell lysates were subjected to immunoblot analysis (top). Densitometric analysis (bottom; mean \pm s.d. $* \mathrm{P}<0.05$ and $* * \mathrm{P}<0.01$ versus control vector). b The cells were transfected with indicated vectors and the expression of E-cadherin was visualized by immunofluorescence. Original magnification, $\times 200 ;$ scale bar, $20 \mu \mathrm{m}$. Representative results were presented from at least three independent experiments with similar results 